\title{
Umbilical Cord Mesenchymal Stem Cells for the Treatment of Atherosclerosis in a Mouse Model: A Randomized Controlled Trial
}

\section{Yijia Song}

Kunming General Hospital of the People's Liberation Army: People's Liberation Army Joint Logistic Support Force 920th Hospital https://orcid.org/0000-0002-2144-3930

\section{Gaomiyang Liu}

Kunming General Hospital of the People's Liberation Army: People's Liberation Army Joint Logistic

Support Force 920th Hospital

\section{Fei Shi}

Kunming General Hospital of the People's Liberation Army: People's Liberation Army Joint Logistic Support Force 920th Hospital

\section{Xiaochun Jiang}

The Third People's Hospital of Yunnan Province

\section{Jufen Liu}

Kunming General Hospital of the People's Liberation Army: People's Liberation Army Joint Logistic Support Force 920th Hospital

\section{Xuejuan Zhang}

Kunming General Hospital of the People's Liberation Army: People's Liberation Army Joint Logistic Support Force 920th Hospital

\section{Qingkeng Lin}

Kunming General Hospital of the People's Liberation Army: People's Liberation Army Joint Logistic

Support Force 920th Hospital

\section{Falian He}

Kunming General Hospital of the People's Liberation Army: People's Liberation Army Joint Logistic Support Force 920th Hospital

\section{Zian Li}

Kunming General Hospital of the People's Liberation Army: People's Liberation Army Joint Logistic Support Force 920th Hospital

\section{Jie He}

Kunming General Hospital of the People's Liberation Army: People's Liberation Army Joint Logistic Support Force 920th Hospital

\section{Guangping Ruan}


Kunming General Hospital of the People's Liberation Army: People's Liberation Army Joint Logistic Support Force 920th Hospital

\section{Rongqing Pang}

Kunming General Hospital of the People's Liberation Army: People's Liberation Army Joint Logistic Support Force 920th Hospital

\section{Xinghua Pan ( $\nabla$ xinghuapan@aliyun.com )}

Kunming General Hospital of the People's Liberation Army: People's Liberation Army Joint Logistic Support Force 920th Hospital

\section{Xiangqing Zhu}

Kunming General Hospital of the People's Liberation Army: People's Liberation Army Joint Logistic Support Force 920th Hospital

\section{Research}

Keywords: Umbilical Cord Mesenchymal Stem Cells, Atherosclerosis, Autophagy, ApoE-/-Mice, Randomized Controlled Trial

Posted Date: February 5th, 2021

DOI: https://doi.org/10.21203/rs.3.rs-170499/v1

License: (c) (i) This work is licensed under a Creative Commons Attribution 4.0 International License. Read Full License 


\section{Abstract}

\section{Background}

The purpose of this study was to determine the efficacy of umbilical cord mesenchymal stem cell (UCMSC) transplantation in a mouse atherosclerosis (AS) model and explore its potential antioxidative stress and lipid metabolism regulatory effects.

\section{Methods}

A mouse AS model was established by feeding $\mathrm{ApoE}^{-/-}$mice a high-fat diet, after which UCMSCs were administered by intraperitoneal injection. The treatment effect was evaluated by measuring heart size and carrying out aortic arch histological staining and blood biochemical detection of blood lipids; RT-PCR was utilized to detect the levels of autophagy-related genes, and Q-TOF was used to detect free fatty acids.

Results

After UCMSC treatment in a mouse model of AS: the number of aortic arch plaques, length of the heart and heart-to-weight ratio were significantly reduced. Aortic arch staining showed that UCMSC treatment reduced the degree of aortic arch intima lesions. Furthermore, serum total cholesterol (TC), triglyceride (TG), and low-density lipoprotein cholesterol (LDL-C) levels were decreased, while the serum high-density lipoprotein cholesterol (HDL-C) level was increased The transcript levels of autophagy-related genes in the heart and liver were significantly higher in AS model mice than in control mice. Finally, the levels of phosphatidylinositol (PI) and ceramide (Cer) in the mouse plasma decreased to the level of the metabolite $\mathrm{PI}$ in the liver.

\section{Conclusions}

UCMSCs increased the overall autophagy level in AS model mice, improved liver metabolic dysfunction, inhibited the formation of atherosclerotic plaques and improved the clinical symptoms of AS in the mouse model.

\section{Introduction}

Atherosclerosis (AS) is a chronic progressive disease involving the large and middle arterial walls with a variety of complex causes[1]. Atherosclerotic cardiovascular disease (ASCVD) based on AS threatens human health and has become the leading cause of human death over the past 10 years[2-4]. At present, the routine treatment of AS in the clinic is mainly based on lipid-lowering drugs, vascular endothelial protective drugs and thrombolytic anticoagulants[5-7]. Various guidelines recommend that lipid-lowering drugs can be used for the primary and secondary prevention of ASCVD events[8], and among these drugs, the related evidence is strongest for statins. On the other hand, debilitating comorbidities, and multiple medications can increase the risks of medication use for certain high-risk ASCVD patients[9]. 
The potential for harm due to existing drug treatments necessitates the identification of new solutions, among which stem cell therapy is undoubtedly attracting worldwide attention. Both bone marrow- and adipose-derived mesenchymal stem cells (MSCs) (BMSCs, and ADMSCs, respectively) have a good therapeutic effect on AS. BMSCs could not only inhibit inflammation but also significantly reduce dyslipidaemia in mice. ADMSCs significantly improved dyslipidaemia in mice and significantly inhibited weight gain in obese mice[10,11]. The method used to obtain BMSCs is invasive, and the number of cells obtained is limited, which limits their clinical application. Umbilical cord mesenchymal stem cells (UCMSCs) are similar to BMSCs and ADMSCs in biological characteristics, such as their morphology, viability, clonogenicity, proliferation and immunophenotype[12]. The advantages of UCMSCs are a noninvasive collection method, small inter-batch differences, capability of large-scale production, and low ethical and legal risks[13, 14]. Therefore, the safety and effectiveness of allograft UCMSCs have been closely monitored[15]. UCMSCs have been applied to animal models of numerous diseases and entered the phase 1 clinical trial stage for the treatment of certain refractory diseases[16]. There have been no reports of long-term adverse reactions, tumour formation or cell rejection upon UCMSC application. The efficacy of UCMSCs in the treatment of some diseases is better than that of BMSCs[17]. Therefore, UCMSCs may provide a new solution for the prevention and treatment of AS and ASCVD.

The tail vein and intraperitoneal injection methods are the two most common routes used to administer factors to mice. Many studies have confirmed the efficacy of MSCs in an AS mouse model. In these studies, the MSCs were transplanted via tail vein infusion. However, there are limitations to this mode of administration. First, tail vein injection is highly difficult; second, the likely aggregation and large volume of UCMSCs can easily cause mice to die due to embolism when UCMSCs enter the mouse circulatory system through the tail vein. In contrast, intraperitoneal injection is easy to perform, the contents of the injection are easily absorbed by the peritoneum, and the animal mortality rate with this method is low. Multiple studies have indicated that intraperitoneal injection is superior to tail vein injection[18-20]. Studies have shown that intraperitoneal injection is safer than intravenous injection and can allow MSCs to migrate to different tissues and home properly[21]. Therefore, use of the intraperitoneal injection route is of great importance for the clinical application of MSCs.

Previous studies have provided much experimental evidence that MSCs not derived from the umbilical cord improve AS symptoms and regulate blood lipids. BMSCs improved AS in ApoE-knockout (KO) mice by exerting an immunomodulatory effect. MSCs inhibited the calcification of vascular smooth muscle cells (VSMCs) by inhibiting injury and apoptosis. Furthermore, induced pluripotent stem cell-derived MSCs (iPSC-MSCs) alleviated atheroma in $\mathrm{ApoE}^{-/-}$mice. Sclerosis reduces the level of inflammatory cytokines, but ADMSCs improve dyslipidaemia and hyperlipidaemia in obese $\mathrm{db} / \mathrm{db}$ mice by regulating protein kinases and lipases[22-25]. However, randomized controlled studies of intraperitoneal UCMSC injection for AS have not been reported.

Unlike other existing studies on the treatment of AS with MSCs, this study focused on evaluating the efficacy of intraperitoneal injection and exploring the mechanism of UCMSC treatment for AS via autophagy and metabolism. We found that in an AS mouse model, UCMSCs inhibited atherosclerotic 
plaque formation, reduced blood lipid levels, and improved autophagy. We used metabolomic methods to analyse the lipidome of model mice, which provided direct laboratory evidence and supplemented a theoretical model of the clinical transformation of UCMSCs in the treatment of AS.

\section{Materials And Methods}

\section{Animals}

Male ApoE ${ }^{-/-}$mice (strain C57BL/6) and C57BL/ 6 mice at 6 weeks of age (Nanjing Junke Biotechnology Co., Ltd., Nanjing China) were used for this study. All animals were raised in specific pathogen-free (SPF) animal laboratories with SPF microorganisms. The animals were maintained in an SPF room at $22^{\circ} \mathrm{C}$ with a 12-hour light/dark cycle and had free access to food and water. All animal experiments were conducted in accordance with the appropriate regulations and had been approved by an ethics committee (2018014).

\section{Induction of AS}

AS was induced by feeding a high-fat diet (Nanjing Junke Biotechnology Co., Ltd., Nanjing China) to 6week-old $\mathrm{Apo}^{-/-}$mice for 6 weeks[26]. Mouse tail DNA was obtained and used to identify the mouse genotype. Three ApoE ${ }^{-/-}$mice fed a high-fat diet and three normal control C57BL/ 6 mice fed a normal diet at the age of 12 weeks were randomly selected for model evaluation. The mice were sacrificed by cervical dislocation to obtain the aortic arch and blood. Movat staining of deparaffinized aortic sections was performed. Samples were placed in 1\% Alcian blue for $20 \mathrm{~min}$, washed with water, and placed in alkaline alcohol at $56^{\circ} \mathrm{C}$ for $10 \mathrm{~min}$. This was followed by serial exposure to the following solutions: OrceinVerhoeff solution, woodstain scarlet-acid fuchsin solution, $0.5 \%$ acetic acid, $5 \%$ phosphotungstic acid, $100 \%$ ethyl alcohol, alcoholic saffron, and finally xylene. Serum was obtained after centrifugation for blood lipid testing.

\section{Isolation and culture of UCMSCs}

The umbilical cord was collected from full-term caesarean section neonates with informed consent from the mother or her family and approval by the ethics committee (2018014). The maternal age was less than 30 years old. Furthermore, each mother was healthy; had no previous history or family genetic history; and tested negative for HIV, syphilis, hepatitis B, and hepatitis C. Additionally, each foetus was not deformed. In this study, UCMSCs were isolated by the adherent culture method[27]. Cells were collected and cultured in complete medium at $37^{\circ} \mathrm{C}$ in a $5 \% \mathrm{CO}_{2}$ atmosphere. The complete medium consisted of 90\% DMEM/F12 (Gibco, USA) and 10\% FBS (Gibco). Cells were passaged when they reached $80 \%-90 \%$ confluence. UCMSCs passaged to the third generation were collected for identification and cell therapy.

\section{Characterization of UCMSCs}


Expression of the cellular markers CD73, CD90, CD105, CD45 and CD34 was measured by flow cytometry analysis as described. UCMSCs were trypsinized and fixed in $4 \%$ paraformaldehyde, re-suspended in PBS $(\mathrm{BI}, \mathrm{USA})$, and then incubated with fluorescently conjugated antibodies against CD73, CD90, CD105, CD45, CD34 or the corresponding isotype controls (BD, USA) at room temperature for $30 \mathrm{~min}$. An LSR flow cytometer was used to analyse the samples. An inverted microscope was used to observe the biological morphology of the UCMSCs. Cells at passage 3 were collected and inoculated into a six-well plate, induced for 21 days in adipogenic differentiation solution, fixed in $4 \%$ formalin for 10 min and stained with oil red $\mathrm{O}$ (Cyagen Biosciences, Suzhou China), after which the formation of lipid droplets was observed under a microscope. Cells of the same generation were seeded in a six-well plate pre-coated with gelatine, and the osteogenic differentiation solution was changed regularly. Twenty-eight days later, alizarin red staining (Cyagen Biosciences, Suzhou China) of the fixed cell samples was performed to observe calcium nodules. Alcian blue staining (Cyagen Biosciences, Suzhou China) of the deparaffinized, 28-day chondrogenic induced cell sections was performed.

\section{UCMSC treatment}

The mice received UCMSC transplantation at 12 weeks of age. Three groups were set in this randomized controlled experiment. The treatment group (AS model mice) was intraperitoneally injected with UCMSCs ( $1 \times 10^{7}$ cells $/ \mathrm{kg}$, at passage 3 , re-suspended in $200 \mu \mathrm{L}$ of saline), and mice in the model control group (AS model mice) and normal control group (normal C57 mice) were intraperitoneally injected with saline (200 $\mu \mathrm{L}$ ). The treatment cycle was 8 weeks, the frequency was once a week, and efficacy was evaluated 4 weeks after the end of treatment.

\section{Dissection and histology}

The mice in each group were sacrificed and dissected at 24 weeks of age to obtain the heart, liver, aortic arch and peripheral blood. The long and short dimensions of the heart were measured with Vernier calipers, and an electronic balance was used to measure the heart weight (after sacrifice) and weight of the mice (before sacrifice). HE staining of dewaxed sections of the heart was performed as follows. Heart tissues were fixed in $4 \%$ paraformaldehyde for $24 \mathrm{~h}$, embedded in paraffin, and sectioned. For HE staining, the slides were immersed in filtered haematoxylin for $6 \mathrm{~min}$, rinsed with water, and stained with eosin for another 1-2 min. Sections were rinsed with water, dehydrated in ascending alcohol solutions, cleared with xylene, and mounted with a cover slip for observation. Oil red 0 staining of dewaxed sections of the aortic arch was performed. Blood serum was obtained by centrifugation (3000 rpm, $10 \mathrm{~min}$, room temperature) and placed in an automatic biochemical analyser for blood lipid testing. The livers were saved in tissue saving solution (TIANGEN, Beijing China) for RNA isolation.

\section{Quantitative real-time PCR}

Total RNA was extracted from the heart and liver tissues and reverse transcribed into cDNA using a RevertAid First Strand cDNA Synthesis Kit (Thermo, USA). The primers were designed by PrimerPrimer5 software. The following primers were used: Atg14囚forward: GAGCATAACAACCCCGCCTA, reverse: 
CTTGCTGAGGTTTTCGCCAC; Atg7: forward: TTGAGCGGCGACAGCATTAG, reverse:

TTAAAGGGGGCGAACTGCAA; Becn1: forward: AGGCATGGAGGGGTCTAAGG, reverse:

GCCTGGGCTGTGGTAAGTAAT; LC3b: forward: AGTCAGATCGTCTGGCTCGG, reverse:

AGCCGGACATCTTCCACTCT; sirt1: forward: CGGCTACCGAGGTCCATATAC, reverse:

AGTCAGGAATCCCACAGGAGA; sirt5: forward: CGGTCCCCACCGCTTTTTG, reverse:

ACACTTCCGAAAGTCTGCCA; and gapdh: forward: CTCTCTGCTCCTCCTGTTCGACAG, reverse:

GTGTAATCATATTGGAACATGTAG. PCR was performed using the CFX96C1000 sequence detection system (Bio-Rad, USA). Fold changes in gene expression were normalized to the expression of gapdh.

The reagent 2× T5 Fast qPCR Mix (SYBR Green I) (TSINKE, Beijing China) was used for real-time quantitative PCR, and the data were analysed and processed with CFX96 Manange software. Three replicate wells were set for each sample. GAPDH was used as the internal reference for relative quantification, and the $2 \otimes \triangle \triangle \mathrm{Ct}$ relative quantification method was used to analyse the expression levels.

\section{Metabonomic sequencing and analysis}

Metabolites were individually extracted from the liver and plasma of the mice, and the supernatant was used for detection. The LC/MS system used for lipidomics analysis was an Agilent 1290 ultra-highperformance liquid phase tandem AB Sciex Triple TOF 6600 high-resolution mass spectrometer. The column used was a Phenomen Kinetex 1.7u C18 100A column (100 x $2.1 \mathrm{~mm}$ ) purchased from Phenomen. The xcms4lipid program developed by the laboratory based on XCMS and a self-built library were used for material identification and data processing. First, the secondary data were screened. As long as the forward or reverse sequence was identified, the peak was retained; peaks from the data were matched according to the $\mathrm{mz}$ tolerance $\pm 25 \mathrm{ppm}$.

\section{Statistical analyses}

All data were analysed by GraphPad 5 statistical software and are shown as the mean \pm standard deviation $(x \pm S D)$. Differences between groups were compared by Student's t-test and ANOVA. Differential metabolite analysis was carried out using multivariate statistical analysis to determine the significance of differences in metabolites, and Kyoto Encyclopedia of Genes and Genomes (KEGG) pathway analysis was used to test the significance of differential metabolite enrichment. $p<0.05$ indicated statistical significance.

\section{Results}

\section{Isolation and characterization of UCMSCs}

Primary UCMSCs migrated from around the tissue block, adhered well to the plastic flask surface and showed a fusiform shape (Sup Fig. 1A); UCMSCs that showed vortex-like growth were removed and mixed with other cells when the confluence reached more than 90\% (Sup Fig. 1B). At passage 3, UCMSCs exhibited a fibroblast-like shape (Sup Fig. 1C). 
The UCMSCs successfully differentiated into adipocytes, osteoblasts, and chondroblasts (Sup Fig. 1D, E, F). Additionally, the UCMSCs differentiated into osteoblasts, which were positively stained with Alizarin red after 21 days of osteoblast induction (Sup Fig. 1D). The dyes bound calcium in the matrix and displayed a red colour. When UCMSCs were in the induction medium, which promoted adipocyte differentiation, lipid droplets gradually accumulated in the UCMSC cytoplasm and were stained with oil red O (Sup Fig. 1E). The differentiation of UCMSCs into chondroblasts was also recorded in vitro after the induction of MSCs for 28 days in induction medium. Overexpression and accumulation of proteoglycans and collagen I in these differentiated cells were evaluated by Alcian blue staining (Sup Fig. 1F).

At passage 3, UCMSCs showed expression of the common MSC markers CD73 (99.8 $\pm 0.35 \%$ ), CD 90 (96.9 $\pm 0.44 \%$ ) and CD105 (92.0 $\pm 0.34 \%$ ) (Sup Fig. 1I, J, K). However, the UCMSCs were negative (or low)

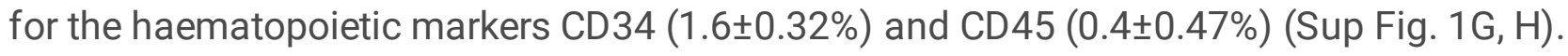

\section{Establishment of a mouse model of AS}

A mouse model of AS was successfully established, and genotype, aortic arch plaque formation, and blood hyperlipidaemia biochemical indicators were identified. Visualization on agarose gels in which lane 1 contained the DNA ladder used to indicate the size of the fragment showed that lanes 2-15 (the model mouse samples) contained 245-bp fragments, while lanes 16 and 17 (control C57 mouse samples) contained 155-bp fragments (Sup Fig. 2A, B). The target band in the wild-type mouse sample was 165 bp, and the band from the KO mice was approximately $245 \mathrm{bp}$, which suggested that mice in the model group in the experiment were $\mathrm{ApoE}^{-/-}$mice.

Movat staining of the aortic arch in the model group and control group suggested that aortic arch plaques had formed in the model group. Movat staining stains plaques red, but normal tissues are not stained. Normal control mice showed no irregular plaque formation in the aortic arch after high-fat diet feeding (Sup Fig. 2C), and irregular plaques appeared in the aortic arches of $\mathrm{ApoE}^{-/}$mice (the plaques were stained red) (Sup Fig. 2D). We also compared the blood lipid levels of the normal control mice and $\mathrm{ApoE}^{-/}$mice (Sup Fig. 2E), which showed that the serum total cholesterol (TC) and serum low-density lipoprotein cholesterol (LDL-C) levels in the $\mathrm{ApoE}^{-1-}$ model mice were $17.19 \pm 0.93 \mathrm{mmol} / \mathrm{L}$ and $12.31 \pm 0.18$ $\mathrm{mmol} / \mathrm{L}$, respectively, which were significantly higher than those in the control group $(p \llbracket 0.01 \llbracket n=3)$.

\section{UCMSC treatment improved cardiac remodelling in mice}

Compared with the model control group, the treatment groups showed a significantly shorter maximum cardiac transverse diameter $(p<0.01, n=5)$ (Fig. 1A) and shorter minimum cardiac transverse diameter. When the heart-to-weight ratio was defined as the ratio of the wet weight of the heart divided by body weight, the heart-to-weight ratio of the treatment group was significantly lower than that of the control group $(p<0.01, \mathrm{n}=5)$ (Fig. 1B). The ventricular wall of the model control group was significantly thicker, as determined b-y HE staining, while the thickness of the ventricular wall of the treatment group was significantly thinner than that of the model control group (Fig. 1C). 


\section{UCMSC treatment reduced aortic arch plaque formation}

Changes in aortic arch plaque were observed after UCMSC treatment. Compared with those in the model control group, the number and area of lipid plaques in the aortic arch inner wall in the treatment group were reduced, and no lipid deposition was observed in the aortic arch inner wall of the normal control group (Fig. 1D). Movat staining was applied as a reflection of the pathological state of the aortic arch plaque. The nuclei and elastic fibres were dyed black, the proteoglycans were dyed blue, the collagen fibres and mesh fibres were dyed yellow, the muscles were dyed red, and the fibrin and red blood cells were dyed bright red. In the model control group, the intima of the aortic arch vessel wall was thickened, foam cells had aggregated, the intima was incomplete, and elastic fibres in the vessel wall were broken. The degree of lesioning in the aortic arch vessel wall was reduced compared with that in the model group, the intima of the aortic arch was partly thickened, and the elastic fibres of the vessel wall had basically returned to normal. In the normal control group, the thickness of the blood vessel wall was uniform, and the intima and elastic fibres of the blood vessel wall were intact (Fig. 1E).

\section{UCMSCs reduced serum lipid levels}

The blood lipid levels of the three groups of mice were detected, which showed that the levels of TG, TC, and LDL-C in the UCMSC treatment group were significantly lower than those in the model control group $(p<0.05, \mathrm{n}=15)$, while the HDL-C level in the UCMSC treatment group was higher than that in the model control group $(p<0.05, \mathrm{n}=15)$ (Fig. 1F).

\section{Changes in the levels of autophagy-related genes}

Studies have shown that the molecular mechanism of AS is related to autophagy. In this study, we speculated that UCMSCs would regulate the metabolism of AS in mice by regulating the level of autophagy and ultimately treat AS. To test this hypothesis, we determined the transcription levels of autophagy-related genes in the heart and liver and found that autophagy-related molecules were significantly upregulated in the treatment group compared with the other two groups and that the overall autophagy level was increased upon treatment (Fig. 2).

\section{Metabolomics analysis}

To clarify how UCMSCs regulate lipid metabolism, the plasma and liver metabolites of the three groups were studied by metabolomics. The metabolic products were sorted in descending order according to the difference multiple, and then products whose secondary matching score was close to 1 were selected (Fig. 3). Hierarchical clustering analysis classified metabolites with similar or complementary characteristics into one group (Fig. 4) and finally screened out the metabolites with statistically significant differences, such as phosphatidylinositol (PI) and ceramide (Cer). In detail, the plasma PI and Cer levels of the treatment group were lower than those of the model control group, while the plasma PI and Cer levels of the model control group were higher than those of the normal control group (Fig. 5); the 
liver PI levels of the treatment group and normal control group were lower than those of the model control group (Fig, 5).

KEGG analysis was used to analyse the metabolic pathways enriched in the differential metabolites in mice to identify the pathways in which all differential metabolites participate. Through enrichment and topology analyses of the pathways that include the differential metabolites, enrichment analysis obtained the $p$ value (Raw $p$ ), and the influence factor (Impact) was obtained from the topology analysis. The pathways were further screened according to $p$ value, and influencing factors were ranked in ascending and descending order, which showed that the key pathways most highly correlated with the differentially abundant metabolites were glycerophospholipid metabolism and linoleic acid metabolism (Fig. 6).

\section{Discussion}

The mechanism of AS formation is currently unclear, but the best studied factor and focus of most domestic and foreign scholars is the relationship between oxidative stress, autophagy and AS[28-30]. This study found that UCMSC treatment significantly changed the transcription levels of autophagyrelated genes in the hearts and livers of AS model mice. The level of autophagy in the model control mice was low, but the level of autophagy increased significantly after UCMSC treatment, which verified the hypothesis that the occurrence and development of AS are related to the level of autophagy. This result is corroborated by related research[31]. Grootaert MOJ found that the pathological process of AS is related to decreased autophagy[32,33]. Oswald $\mathrm{J}$ reported that MSCs can home to the damaged site in vitro and then differentiate into endothelial cells to repair damaged blood vessels[34]. The evidence that MSCs can differentiate into cells at the site of injury after intravenous injection into the body is not sufficient, but an increasing number of studies have confirmed that MSCs secrete exosomes to regulate immune and metabolic functions to repair damaged blood vessels and organs[35, 36]. Our group's previous study reported that UCMSCs secrete exosomes to upregulate autophagy and protect cells from reactive oxygen species (ROS) damage[37]. UCMSCs were suggested to secrete exosomes to improve the level of heart and liver autophagy and repair tissue damage (including endothelial damage) in AS model mice, i.e., the therapeutic effects of UCMSCs in AS mainly depends on exosome-dependent environmental regulation rather than cell differentiation.

Lipidomic analysis of the mouse liver and serum found that UCMSC treatment reversed changes in PI and Cer levels in the liver and plasma of AS model mice. MSCs can improve the formation of arterial plaques and reduce the levels of TC, TG, and LDL-C in the serum, significantly improving AS, which is consistent with our results[10, 24, 38, 39]. However, the results of metabolomics studies on UCMSC treatment in AS models have not been reported. The metabolomics data in this study showed that the plasma PI and Cer levels of AS model mice decreased after UCMSC treatment, while changes in the level of only $\mathrm{PI}$ in the liver were reversed. The results of this experiment have not been reported at home or abroad supplementing experimental evidence for the use of UCMSCs in the treatment of AS and 
providing ideas for subsequent in-depth study of the anti-AS effects of UCMSCs from a metabolism perspective.

Whether in the liver or in plasma, the PI level of the treatment group was significantly decreased. PI can be activated by phosphatidylinositol kinase to produce phosphatidylinositol phosphate (PIP) and phosphatidylinositol diphosphate (PIP2), which can be hydrolysed to inositol triphosphate by phospholipase C (PLC) on the cell membrane (IP3) and diacylglycerol (DAG) [40]. IP3 can be used as a substrate for PI3K participation in the PI3K-AKT signalling pathway, which has been shown to be closely related to the regulation of cellular oxidative stress and autophagy levels[41]. Serum-free fatty acid, cholesterol, and glycerine levels in model mice in which the PI3K catalytic subunit had been specifically knocked out were significantly reduced[42]. When pten, an important negative regulator of the PI3K-AKT pathway, was knocked out, mice exhibited fatty liver, and plasma free fatty acid levels increased significantly[43]. When the PI3K-AKT pathway was inhibited, the expression of nlrp3 and production of ROS were reduced, the activation of nlrp3 inflammatory bodies was inhibited, and the levels of downstream IP-1 $\beta$ and IP-18 were downregulated[44, 45]. Based on these results, we hypothesized that UCMSCs can activate the PI3K-AKT signalling pathway, downregulate the level of $\mathrm{PI}$, upregulate the expression of autophagy-related genes, and relieve ROS damage to endothelial cells, thus relieving AS symptoms or even curing AS. The related regulatory mechanism needs further research and future confirmation.

A key event in the formation of AS plaques is the accumulation of atherosclerotic lipoproteins, which increases the lipoprotein content in foam cells. Sphingomyelin is hydrolysed by sphingomyelinase to generate Cer and phosphocholine, a link to the initiation of lipoprotein aggregation. Sphingomyelin is transported by atherosclerotic lipoproteins to the arterial wall, and Cer is produced by sphingomyelinase in the arterial wall, promoting lipoprotein aggregation[46]. A study examined the Cer content in plaques in 200 patients with carotid AS and found that the Cer level was significantly increased, which was related to inflammatory cytokines and caspase- 3 in plaques, and could induce the inflammatory reaction in human coronary artery smooth muscle cells[47]. In addition, Cer is associated with histological markers of unstable plaque. We found that an increase in Cer was accompanied by disorder of the aorta and aortic arch structure and increased plaque formation. Similar reports have indicated that the Cer content in the serum and aorta in type 2 diabetes model rats was significantly increased; furthermore, in type 2 diabetes, the endothelium-dependent vasodilation function of diabetes model rats was decreased, and the endothelial cell structure was disordered[40]. Combined with the relevant research results, the increase in Cer level was suggested to be related to disorder in vascular endothelial cell structuring and the formation of AS plaques, which may become a therapeutic target for AS. UCMSC treatment significantly reduced the level of Cer in plasma. We simultaneously observed other indicators in AS model rats: heart size; the number of plaques that formed in the aortic arch; and the levels of TC, TG, and LDL-C in plasma. UCMSCs are feasible as a drug for the treatment or prevention of AS.

\section{Conclusions}


In summary, our results showed that UCMSCs could significantly improve AS in $\mathrm{ApoE}^{-/-}$mice, potentially through the downregulation of PI and Cer, which activated or inhibited PI3K-AKT or other related signalling pathways in vascular endothelial cells, smooth muscle cells and other cells. Multiple functions of these cells, including apoptosis, autophagy, and oxidative stress, were regulated, improving disorder in vascular endothelial cell deconstruction and AS plaque formation. The specific mechanisms of these adjustments need to be further studied and clarified in future experiments.

\section{Abbreviations}

\begin{tabular}{ll} 
AS & atherosclerosis \\
\hline Cer & ceramide \\
\hline DMSO & dimethyl sulfoxide \\
\hline FBS & fetal bovine serum \\
\hline HDL-C & high density lipoprotein-cholesterol \\
\hline LDL-C & low density lipoprotein-cholesterol \\
\hline PBS & phosphate buffered saline \\
\hline PCR & polymerase Chain Reaction \\
\hline PI & phosphatidylinositol \\
\hline RNA & ribonucleic acid \\
\hline TC & total Cholesterol \\
\hline TG & triglyceride \\
\hline UCMSC & umbilical cord mesenchymal stem cells
\end{tabular}

\section{Declarations}

\section{Ethical approval and consent to participate}

Experimental protocols were approved by the Experimental Animal Ethics Committee of the 920th Hospital of the PLA Joint Logistics Support Force. The approval number: 2018014

\section{Consent for publication}

Not applicable.

\section{Availability of data and material}

All data generated or analysed during this study are included in this published article. 


\section{Competing interests}

The authors declare that they have no competing interests.

\section{Funding}

This work was supported by grants from the Yunnan Science and Technology Plan Project Major Science and Technology Project (2018ZF007)

\section{Authors' contributions}

YJS, GMYL and XHP designed the study. YJS, JFL, FLH, ZAL, JH and XCJ carried out experiments. YJS, RQP, XJZ, QKL and XHP analyzed the data. YJS, GMYL, XHP and XCJ drafted and revised the paper. All authors read and approved the final manuscript for publication.

\section{Acknowledgements}

We thank Freescience's Experts for assisting with the preparation of this manuscript.

\section{References}

1. Lusis, A.J., Atherosclerosis. Nature, 2000. 407(6801): p. 233-41.

2. Yang, X., et al., Predicting the 10-Year Risks of Atherosclerotic Cardiovascular Disease in Chinese Population: The China-PAR Project (Prediction for ASCVD Risk in China). Circulation, 2016. 134(19): p. 1430-1440.

3. Wang, $\mathrm{H}_{\text {., }}$ et al., Comparisons of the Framingham and ASCVD risk scores for coronary heart disease risk prediction in Chinese men. Int J Cardiol, 2018. 266: p. 269.

4. Rosenblit, P.D., Extreme Atherosclerotic Cardiovascular Disease (ASCVD) Risk Recognition. Curr Diab Rep, 2019. 19(8): p. 61.

5. Last, A.R., J.D. Ference, and E.R. Menzel, Hyperlipidemia: Drugs for Cardiovascular Risk Reduction in Adults. Am Fam Physician, 2017. 95(2): p. 78-87.

6. Zuo, Y., et al., The role of cardiovascular disease risk assessed by ASCVD score in primary thrombosis prophylaxis strategy among antiphospholipid antibody carriers. Lupus, 2018. 27(13): p. 2177-2178.

7. Yusuf, S., et al., Effects of an angiotensin-converting-enzyme inhibitor, ramipril, on cardiovascular events in high-risk patients. N Engl J Med, 2000. 342(3): p. 145-53.

8. Koenigsfeld, C., et al., Retrospective Evaluation of ASCVD Risk and Statin Therapy Need in Nondiabetic Patients Based on the 2013 ACC/AHA Cholesterol Guidelines. J Pharm Pract, 2017. 30(3): p. 300-305.

9. Garg, N., et al., Comparison of different cardiovascular risk score calculators for cardiovascular risk prediction and guideline recommended statin uses. Indian Heart J, 2017. 69(4): p. 458-463. 
10. Frodermann, V., et al., Mesenchymal Stem Cells Reduce Murine Atherosclerosis Development. Sci Rep, 2015. 5: p. 15559.

11. Takafuji, Y., et al., Humoral factors secreted from adipose tissue-derived mesenchymal stem cells ameliorate atherosclerosis in Ldlr-/-mice. Cardiovasc Res, 2019. 115(6): p. 1041-1051.

12. Fabre, H., et al., Characterization of Different Sources of Human MSCs Expanded in Serum-Free Conditions with Quantification of Chondrogenic Induction in 3D. Stem Cells Int, 2019. 2019: p. 2186728.

13. Jin, H.J., et al., Comparative analysis of human mesenchymal stem cells from bone marrow, adipose tissue, and umbilical cord blood as sources of cell therapy. Int J Mol Sci, 2013. 14(9): p. 17986-8001.

14. Kern, S., et al., Comparative analysis of mesenchymal stem cells from bone marrow, umbilical cord blood, or adipose tissue. Stem Cells, 2006. 24(5): p. 1294-301.

15. Can, A., F.T. Celikkan, and O. Cinar, Umbilical cord mesenchymal stromal cell transplantations: $A$ systemic analysis of clinical trials. Cytotherapy, 2017. 19(12): p. 1351-1382.

16. Bartolucci, J., et al., Safety and Efficacy of the Intravenous Infusion of Umbilical Cord Mesenchymal Stem Cells in Patients With Heart Failure: A Phase 1/2 Randomized Controlled Trial (RIMECARD Trial [Randomized Clinical Trial of Intravenous Infusion Umbilical Cord Mesenchymal Stem Cells on Cardiopathy]). Circ Res, 2017. 121(10): p. 1192-1204.

17. Zhang, Q., et al., Comparison of therapeutic effects of different mesenchymal stem cells on rheumatoid arthritis in mice. PeerJ, 2019. 7: p. e7023.

18. Nam, Y., et al., Intraperitoneal infusion of mesenchymal stem cell attenuates severity of collagen antibody induced arthritis. PLoS One, 2018. 13(6): p. e0198740.

19. Yousefi, F., et al., Comparison of in vivo immunomodulatory effects of intravenous and intraperitoneal administration of adipose-tissue mesenchymal stem cells in experimental autoimmune encephalomyelitis (EAE). Int Immunopharmacol, 2013. 17(3): p. 608-16.

20. Bastug, F., et al., Compare the effects of intravenous and intraperitoneal mesenchymal stem cell transplantation on ultrafiltration failure in a rat model of chronic peritoneal dialysis. Ren Fail, 2014. 36(9): p. 1428-35.

21. Ullah, M., D.D. Liu, and A.S. Thakor, Mesenchymal Stromal Cell Homing: Mechanisms and Strategies for Improvement. iScience, 2019. 15: p. 421-438.

22. Wang, S., et al., The Bioactive Substance Secreted by MSC Retards Mouse Aortic Vascular Smooth Muscle Cells Calcification. Biomed Res Int, 2018. 2018: p. 6053567.

23. Shi, $\mathrm{H}$., et al., Human induced pluripotent stem cellderived mesenchymal stem cells alleviate atherosclerosis by modulating inflammatory responses. Mol Med Rep, 2018. 17(1): p. 1461-1468.

24. Liu, G.Y., et al., Adipose-Derived Mesenchymal Stem Cells Ameliorate Lipid Metabolic Disturbance in Mice. Stem Cells Transl Med, 2016. 5(9): p. 1162-70.

25. Wang, Z.X., et al., Mesenchymal stem cells alleviate atherosclerosis by elevating number and function of CD4(+)CD25 (+)FOXP3 (+) regulatory T-cells and inhibiting macrophage foam cell 
formation. Mol Cell Biochem, 2015. 400(1-2): p. 163-72.

26. Emini Veseli, B., et al., Animal models of atherosclerosis. Eur J Pharmacol, 2017. 816: p. 3-13.

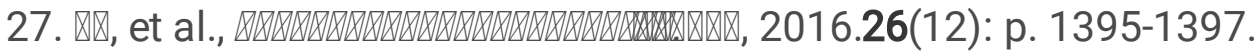

28. Forstermann, U., N. Xia, and H. Li, Roles of Vascular Oxidative Stress and Nitric Oxide in the Pathogenesis of Atherosclerosis. Circ Res, 2017. 120(4): p. 713-735.

29. Bryk, D., W. Olejarz, and D. Zapolska-Downar, The role of oxidative stress and NADPH oxidase in the pathogenesis of atherosclerosis. Postepy Hig Med Dosw (Online), 2017. 71(0): p. 57-68.

30. Di Marco, E., et al., Pharmacological inhibition of NOX reduces atherosclerotic lesions, vascular ROS and immune-inflammatory responses in diabetic Apoe(-/-) mice. Diabetologia, 2014. 57(3): p. 633-42.

31. Gray, S.P., et al., Combined NOX1/4 inhibition with GKT137831 in mice provides dose-dependent reno- and atheroprotection even in established micro- and macrovascular disease. Diabetologia, 2017. 60(5): p. 927-937.

32. Grootaert, M.O.J., et al., Defective Autophagy in Atherosclerosis: To Die or to Senesce? Oxid Med Cell Longev, 2018. 2018: p. 7687083.

33. Grootaert, M.O.J., et al., Vascular smooth muscle cell death, autophagy and senescence in atherosclerosis. Cardiovasc Res, 2018. 114(4): p. 622-634.

34. Oswald, J., et al., Mesenchymal stem cells can be differentiated into endothelial cells in vitro. Stem Cells, 2004. 22(3): p. 377-84.

35. Yu, B., et al., Cardiomyocyte protection by GATA-4 gene engineered mesenchymal stem cells is partially mediated by translocation of miR-221 in microvesicles. PLoS One, 2013. 8(8): p. e73304.

36. Ibrahim, A.G., K. Cheng, and E. Marban, Exosomes as critical agents of cardiac regeneration triggered by cell therapy. Stem Cell Reports, 2014. 2(5): p. 606-19.

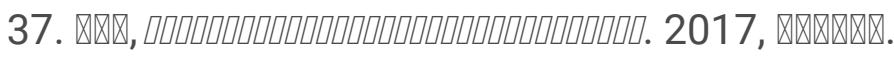

38. Li, F., X. Guo, and S.Y. Chen, Function and Therapeutic Potential of Mesenchymal Stem Cells in Atherosclerosis. Front Cardiovasc Med, 2017. 4: p. 32.

39. Lin, Y.L., et al., Mesenchymal Stem Cells Ameliorate Atherosclerotic Lesions via Restoring Endothelial Function. Stem Cells Transl Med, 2015. 4(1): p. 44-55.

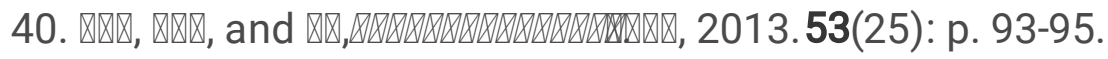

41. Meng, J., et al., EGCG protects vascular endothelial cells from oxidative stress-induced damage by targeting the autophagy-dependent PI3K-AKT-mTOR pathway. Ann Transl Med, 2020. 8(5): p. 200.

42. Sopasakis, V.R., et al., Specific roles of the p110alpha isoform of phosphatidylinsositol 3-kinase in hepatic insulin signaling and metabolic regulation. Cell Metab, 2010. 11(3): p. 220-30.

43. Stiles, B., et al., Liver-specific deletion of negative regulator Pten results in fatty liver and insulin hypersensitivity [corrected]. Proc Natl Acad Sci U S A, 2004. 101(7): p. 2082-7.

44. Fortin, C.F., et al., A class IA PI3K controls inflammatory cytokine production in human neutrophils. Eur J Immunol, 2011. 41(6): p. 1709-19. 
45. Boyle, K.B., et al., Class IA phosphoinositide 3-kinase beta and delta regulate neutrophil oxidase activation in response to Aspergillus fumigatus hyphae. J Immunol, 2011. 186(5): p. 2978-89.

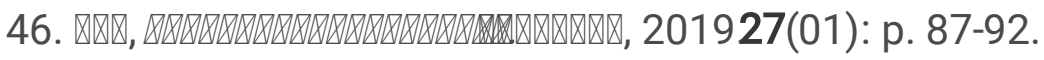

47. Edsfeldt, A., et al., Sphingolipids Contribute to Human Atherosclerotic Plaque Inflammation. Arterioscler Thromb Vasc Biol, 2016. 36(6): p. 1132-40.

\section{Figures}



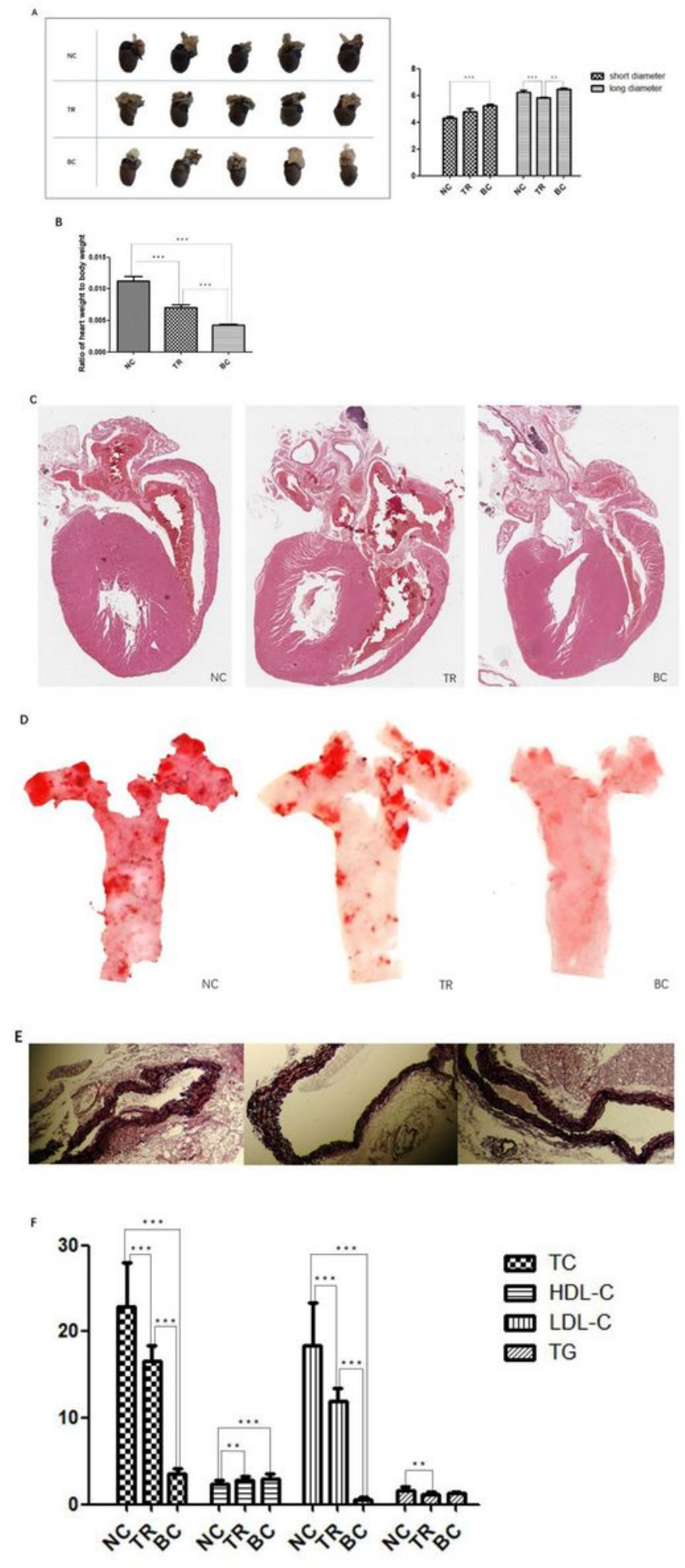

Figure 1

A shows a comparison of heart size between the model control group and normal control group after 4 weeks of UCMSC treatment in AS mice $(n=5)$. B shows the ratio of the wet heart weight to the body weight of mice in the model control group and normal control group $(n=5)$. C shows HE staining of the hearts of AS mice in the model control group and normal control group (40x). D shows oil red 0 staining of the aortic arches of AS mice in the normal control group and model control group (100x). E shows 
Movat staining of the aortic arches of AS mice in the normal control group and model control group (200x). F shows the TG, TC, HDL-C, and LDL-C levels in mice in the model control group and normal control group, NC represents the model control group, TR represents the treatment group, BC represents the normal control group, $* *$ indicates a difference with $p<0.05, * \star *$ indicates a significant difference with $p<0.01, n=15$.
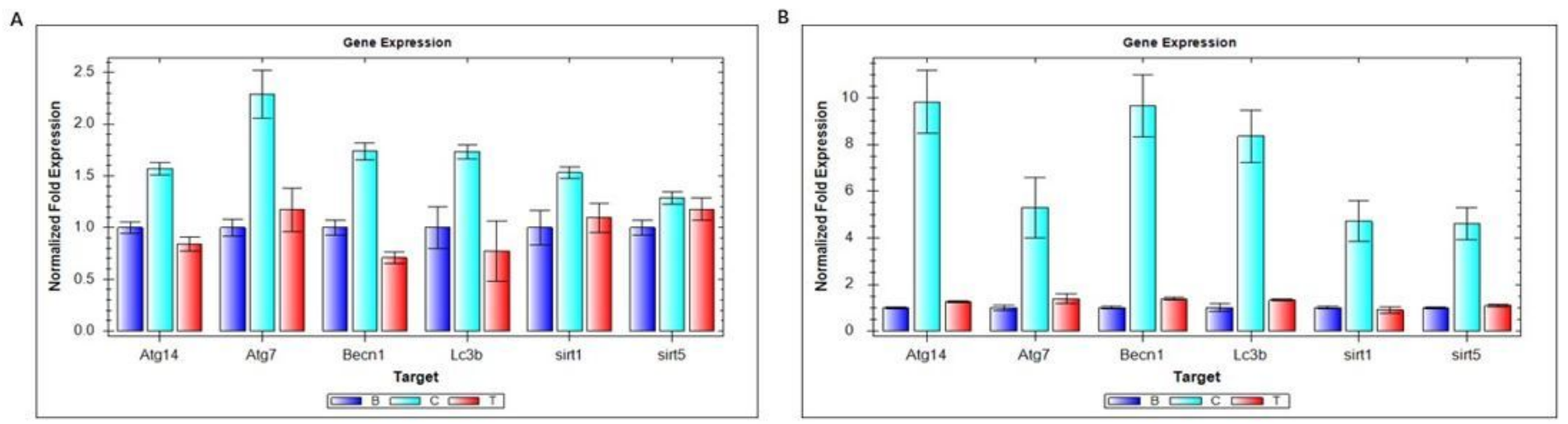

\section{Figure 2}

A shows the transcription levels of genes related to cardiac autophagy. B shows the transcription levels of genes related to liver autophagy (group $B$ is the normal control group, group $C$ is the model control group, group $\mathrm{T}$ is the treatment group, $\mathrm{n}=10$ ).

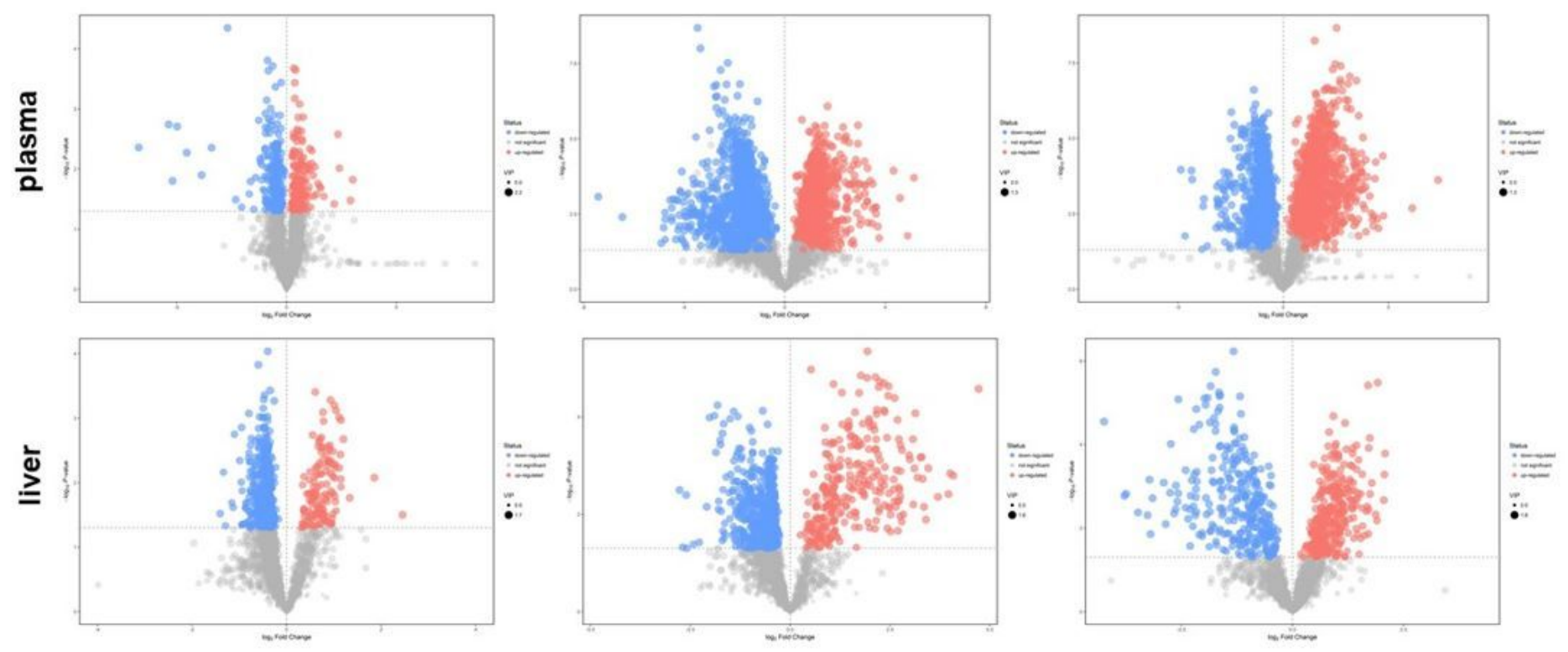

\section{Figure 3}

each dot in the figure represents a metabolite, and the abscissa shows changes in the group contrast ratio of each material ordinate by $\mathrm{p}$ value according to the t-test. The scatter plot shows the OPLS-DA model and the size of the VIP value; a more intense scatter VIP value colour indicates final selection. Metabolites whose abundance was significantly increased are shown in red, metabolites whose 
abundance was significantly decreased are shown in blue, and metabolites whose abundance was not significant different are shown in grey.

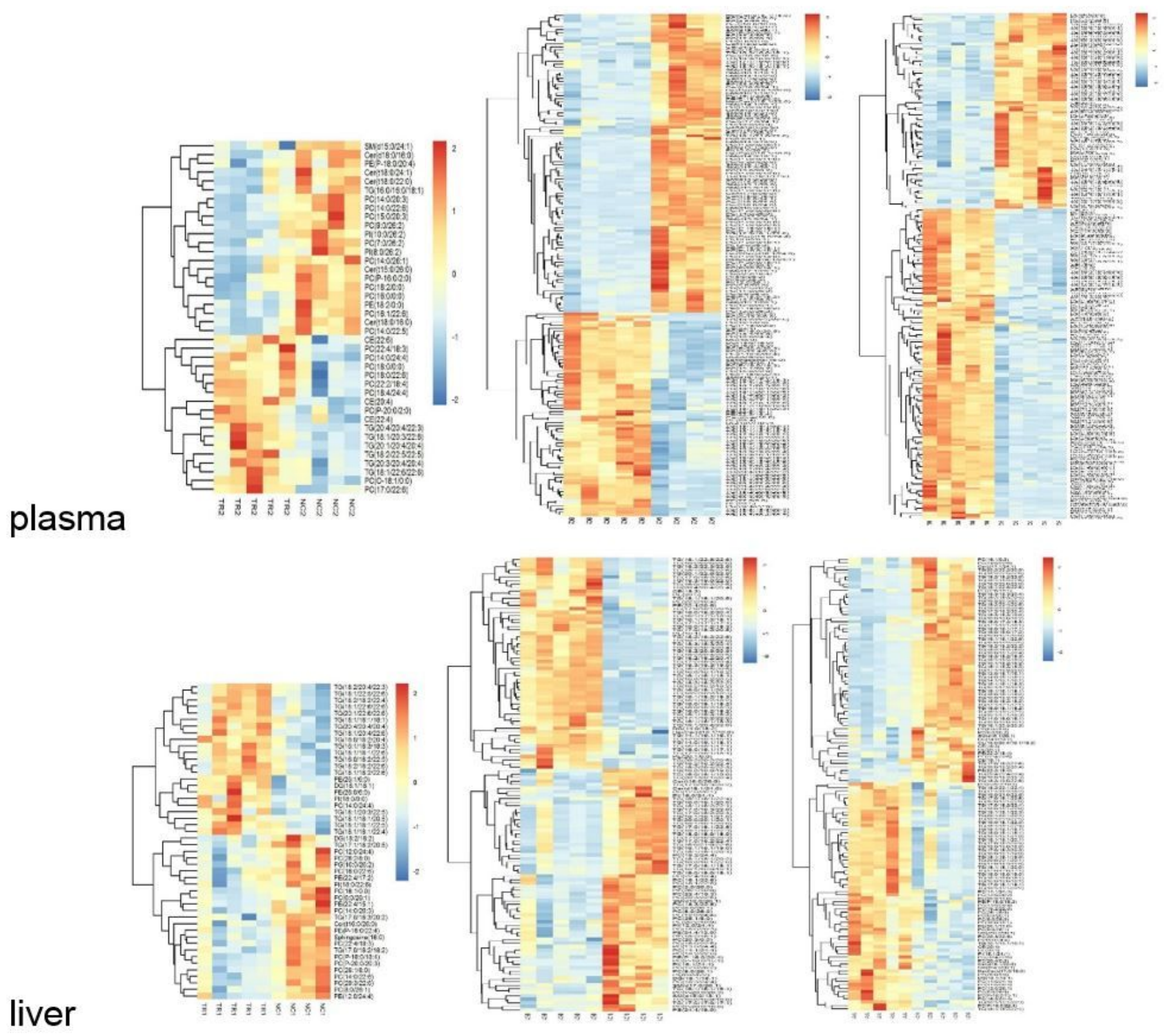

Figure 4

the horizontal axis represents the different experimental groups, the vertical axis represents the differentially abundant metabolites compared with the group, and the colour blocks at different positions represent the relative levels of metabolites at corresponding positions. 

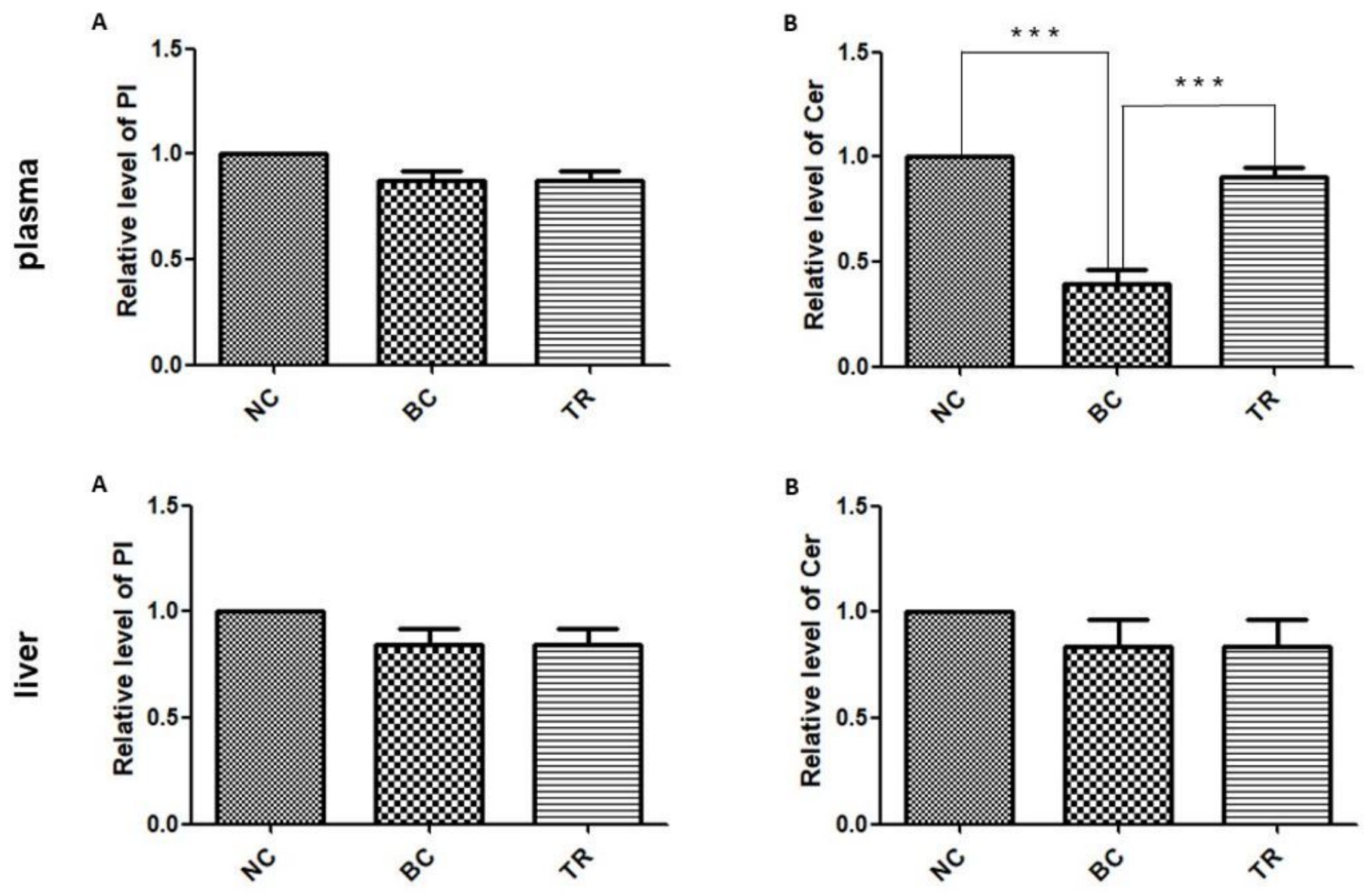

Figure 5

A shows the change in PI level in mice in the treatment group, normal control group and model group. B shows the change in Cer level in mice in the treatment group, normal control group and model group. NC represents the model control group $(n=4), B C$ represents the normal control group $(n=5)$, TR represents the treatment group $(n=5)$, and $\star \star \star$ indicates a significant difference, $p<0.01$. 

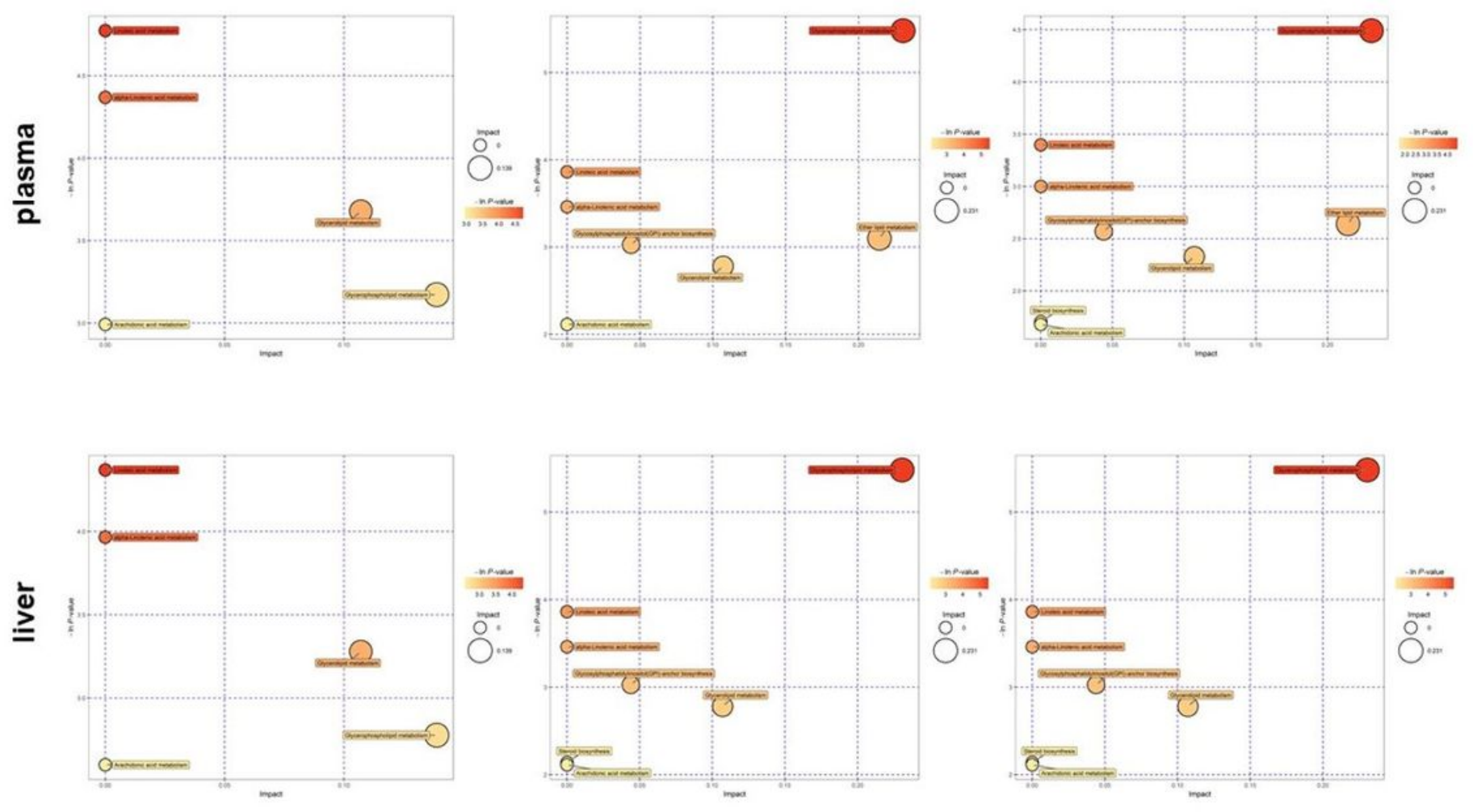

\section{Figure 6}

A is a pathway analysis diagram comparing the treatment group and model control group; $\mathrm{B}$ is a pathway analysis diagram comparing the normal control group and model control group; $\mathrm{C}$ is a pathway analysis diagram comparing the treatment group and normal control group. The results of metabolic pathway analysis are presented as a bubble graph. In the bubble diagram, each bubble represents a metabolic pathway. The x-coordinate and the size of each bubble represent the extent to which the pathway identified by topological analysis was enriched in the influencing factor. The larger the size is, the greater the enrichment in the influencing factor. The y-coordinate of the bubble and the colour of the bubble represent the $p$ value obtained by enrichment analysis. The darker the colour is, the smaller the $p$ value is, and the more significant the degree of enrichment. 